\title{
High Resolution XMM-Newton Data of Nova Outbursts using Warm \& Hot Absorber Models
}

\author{
Ş. Balman and Y. Pekön \\ Middle East Technical University, Dept. of Physics, Inönü Bulvarı, Ankara, Turkey, 06531 \\ email: solen@astroa.physics.metu.edu.tr, yakup@astroa.physics.metu.edu.tr
}

\begin{abstract}
We reanalyze a selection of the XMM-Newton RGS (Reflection Grating Spectrometer) data of two classical novae; V4743 Sgr and V2491 Cyg. The high resolution nova spectra in the X-ray wavelengths show existence of absorption features (e.g., blue shifted). Our main aim is to model the absorption components detected in the high resolution spectra independently from the assumed continuum model. We assume that there is complex absorption of interstellar, photospheric, and of collisionally and/or photoionized gas origin in the moving material along the line of sight from a nova wind or ejecta. In addition to deriving the absorption properties, we obtain CNO abundances of elements in the ejecta/nova wind.
\end{abstract}

Keywords. stars: novae, cataclysmic variables, winds, outflows, abundances - X-rays: stars

\section{Introduction}

Nova 4743 Sgr was discovered in September 2002 (Haseda et al. 2002). It is a very fast nova with large velocities on the order of 2,400 $\mathrm{km} \mathrm{s}^{-1}$ (Kato et al. 2002). Nova 2491 Cyg was discovered in April 2008 (Nakano et al. 2008) and detected to be a very fast nova. Optical spectroscopy reveals large expansion velocities $\left(\sim 4,000-6,000 \mathrm{~km} \mathrm{~s}^{-1}\right.$; Lynch et al. 2008) . Both of the novae we study show a hard X-ray and a soft X-ray component during the outburst stage (V4743 Sgr: Ness et al. 2003, Rauch et al. 2010; V2491 Cyg: Page et al. 2010).

V2491 Cyg was observed (OBSID=0552270501) for an exposure of 39 ks in May 2008; V4743 Sgr was observed (OBSID=0127720501) for an exposure of $35 \mathrm{ks}$ in April 2003 by XMM-Newton. We utilize the RGS data to obtain the high resolution spectra for our analysis. For the analysis, we reprocessed the data and extracted the spectra using the XMM-SAS routine RGSPROC. We used two absorption models within the SPEX software (Kaastra et al. 1996) to model our data along with a simple blackbody emission component for the continuum. One of the models we used in our analysis is the XABS model of SPEX, which calculates the absorption by a photoionized thin slab composed of different ions, located between the ionizing source and the observer. For modeling the column densities of the photoionized gas, we used the CLOUDY software (Ferland et al. 1998). The second absorber model assumes a collisionally ionized (in equilibrium) absorber model instead of the photo-ionized absorber model.

\section{Results \& Discussion}

In order to model the complex absorption, we utilize a photo-ionized warm absorber model (XABS) and a hot, collisionally ionized absorber model (HOT). For simplicity and ease of fitting procedures, we use a blackbody to model the continuum; thus we caution that we are missing some photospheric absorption features. We find blackbody temperatures that are almost the same as the expanding NLTE atmosphere model temperatures 

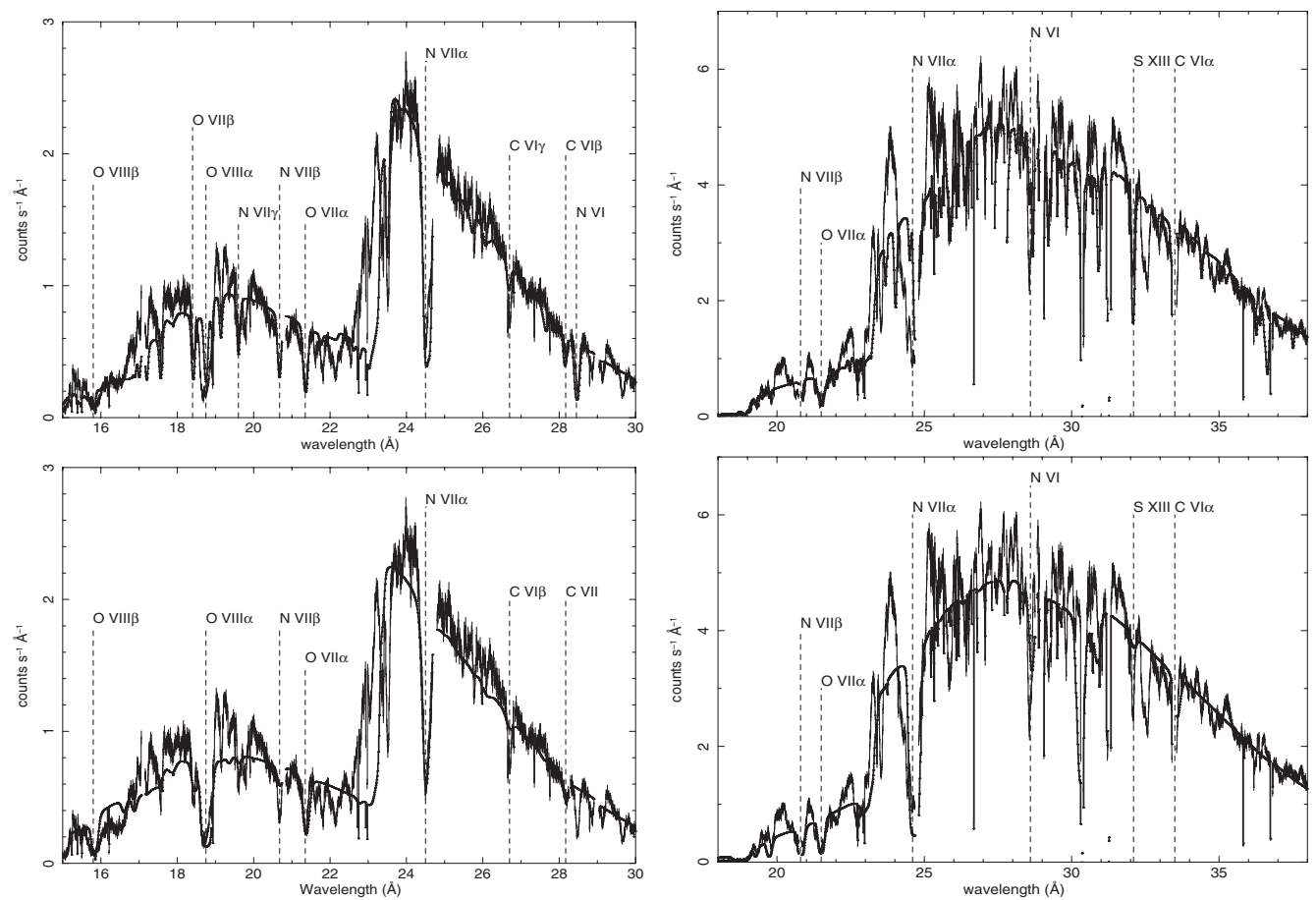

Figure 1. The RGS spectrum of V2491 Cyg fitted with HOTABS (top) and XABS (bottom) models are on the left-hand-side, and the spectrum of V4743 Sgr fitted with the same models are on the right-hand-side. Certain detected blue-shifted absorption lines are labeled.

(for the underlying WD photospheric temperature). Our fits model the ionized absorption features simultaneously, thus calculating a global velocity shift for the absorption component in the data originating from the nova wind/ejecta. We derive a blue-shifted absorber with 3,080-3450 $\mathrm{km} \mathrm{s}^{-1}$ for V2491 Cyg and 1,090-1,600 $\mathrm{km} \mathrm{s}^{-1}$ for V4743 Sgr, which are consistent with the ejecta/wind speeds. We derive CNO abundances from our fits. V2491 Cyg has a nitrogen overabundance of $\mathrm{N}=14-36$ (ratio to solar abundance) where $\mathrm{C}$ and $\mathrm{O}$ are about twice their solar abundance. V4743 Sgr shows a typical signature of H-burning with under-abundant carbon $\mathrm{C}=0.004-0.2$, and enhanced nitrogen $\mathrm{N}=12-53$ and oxygen $\mathrm{O}=24-53$ (all ratios to solar abundances). We find the equivalent hydrogen column density of the ionized absorbers as $(8.0-0.3) \times 10^{22} \mathrm{~cm}^{-2}$ for V2491 Cyg and (3.6-4.3) $\times 10^{23} \mathrm{~cm}^{-2}$ for V4743 Sgr. We suggest that the RGS data of V2491 Cyg is in accordance with a collisionally ionized absorber (e.g., shocks within winds) based on modeling of the major blue-shifted absorption features. However, for V4743 Sgr, we suggest that the photo-ionized warm absorber (e.g., photo-ionized wind) is a more physically consistent interpretation with relatively better $\chi_{\nu}^{2}$ values.

\section{References}

Ferland, G. J. et al. 1998, PASP, 110, 761

Kaastra, J. S. et al. 1996, A\&A, 314, 547

Kato, T. et al. 2002, IAU Circ., 7976

Lynch, D. K. et al. 2008, AJ, 136, 1815

Nakano et al. 2008, IAU Circ., 8934

Ness J.-U. et al. 2003, ApJ, 594, L127

Page, K. L. et al. 2010, MNRAS, 401, 121

Rauch, T., Orio M., Gonzales-Riestra R., Nelson T., Still M., et al. 2010, ApJ, 717, 363 